\title{
Mimicking Amyotrophic Lateral Sclerosis: A Case of a Spinal Dural Arteriovenous Fistula
}

\author{
Mónica Santos $^{a}$ Sofia Reimão ${ }^{b, c}$ Mamede de Carvalho ${ }^{a, c}$ \\ aDepartment of Neurosciences and Mental Health, Neurology, Hospital Santa Maria/ \\ Centro Hospitalar Universitário Lisboa-Norte, Lisbon, Portugal; 'bepartment of Neurology \\ Imagiology, Hospital Santa Maria/Centro Hospitalar Lisboa Norte, Lisbon, Portugal; 'Faculdade \\ de Medicina-Instituto de Medicina Molecular, Universidade de Lisboa, Lisbon, Portugal
}

\section{Keywords}

Amyotrophic lateral sclerosis · Spinal dural arteriovenous fistula · Mimic

\begin{abstract}
A number of conditions can mimic amyotrophic lateral sclerosis (ALS), which are in general excluded by neurophysiological and neuroimaging investigation. We present a novel mimicking disorder. A 58-year-old male, without relevant past medical history, presented with a 7-year history of progressive paraparesis. On examination, he had bilateral thigh atrophy, fasciculations, and asymmetric paraparesis (severe on the left side). Upper motor neuron signs were present in the lower limbs, with normal sensory examination. Needle EMG disclosed mild chronic neurogenic changes in the lower limbs. Brain and spinal cord neuroimaging was normal, namely, in the dorso-lumbar segment. Lumbar puncture showed mild hyperproteinorachia. Diagnosis of slowly progressive (possible) ALS was established. One year later, he required a bilateral support to walk, and neurological examination revealed weak tendon reflexes, abnormal pinprick, and proprioceptive sensation in the legs. Repeated lumbar MRI showed an extensive spinal cord oedema from $\mathrm{T} 7$ to the conus with multiple perimedullary vessel flow voids suggestive of a vascular malformation. Conventional angiography revealed a spinal dural arteriovenous fistula in L2-L3 with the left L4 lumbar branch as the afferent artery. Dural arteriovenous fistula is the most common vascular malformation of the spinal cord, despite being rare. It leads to arterialization of spinal veins, causing venous hypertension, spinal cord oedema, and ischaemia. The clinical picture includes a stepwise, sometimes fluctuant, myeloradiculopathy. In this case, EMG changes did not meet Awaji criteria. This case reinforces the need to critically follow atypical cases to ascertain clinical progression in patients with suspected ALS.
\end{abstract}




\section{Introduction}

Spinal dural arteriovenous fistula (SDAVF) is rare and often misdiagnosed [1], but is still the most common spinal cord vascular malformation. We present the clinical case of a patient with SDAVF mimicking amyotrophic lateral sclerosis (ALS). This is a very important differential diagnosis to consider in atypical ALS since it can be treated with great clinical improvement.

\section{Case Report/Case Presentation}

A 58-year-old male was referred to the ALS clinic in 2017, with a history of progressive lower limb weakness. He first complained of distal weakness of the left lower limb since 2011, which progressed proximally over 3 years and later to the contralateral leg. At this point, there were no sensory complaints, sphincter or erectile dysfunction, cognitive changes, involvement of upper limbs, and bulbar or respiratory symptoms. Past personal and family history was unremarkable.

On examination, he had bilateral thigh atrophy, with fasciculations on the right thigh. Fasciculations were observed in the right triceps as well. Strength testing (Medical Research Council - MRC grading) disclosed asymmetric paraparesis: hip flexion grade 2 bilaterally, knee flexion grade $4+$ on the right and 5 on the left, knee extension grade $4+$ on the right and 5 on the left, ankle dorsiflexion grade $4+$ on the right and 4 on the left, and hallux extension grade $4+$ on the right and 4 on the left. Deep tendon reflexes were found brisk in the 4 limbs. The Babinski sign was present bilaterally, and the left ankle showed clonus. No spasticity was found. Upper limb strength was normal, as well as cranial nerve testing and sensory modalities. The tongue showed no atrophy or fasciculations. Masseter reflex was weak.

Nerve conduction studies showed moderately increased F-waves latency in lower limbs (peroneal and tibialis posterior), but motor and sensory nerve conduction studies were normal. Needle electromyography disclosed mild chronic neurogenic changes in lower limb muscles (vastus medialis and tibialis anterior on both sides) with rare fasciculation potentials. Upper limb muscles (first dorsal interosseous, bilaterally) were normal, but rare fasciculation potentials were detected is distal muscles. The brain and spinal cord (cervical, thoracic, and lumbo-sacral segments) were examined, but no change was found. Lumbar puncture revealed mild hyperproteinorachia $(61 \mathrm{mg} / \mathrm{dL})$, with normal cells $\left(1.2\right.$ cells $\left./ \mathrm{mm}^{3}\right)$ and glucose levels, and negative serology for Lyme disease and syphilis. Creatinine kinase levels were normal.

In the presence of progressive asymmetric paraparesis in a middle-aged man, with both upper and lower motor neuron signs in one single region, and no evidence of other disease, a diagnosis of possible slowly progressive ALS was considered [2]. The patient declined more investigations, in particular MRI, due to claustrophobia.

The patient continued to progress clinically. In 2019, he required bilateral support to walk and presented with new complaints: sensation of numbness in lower limbs, urinary urgency, and anal sphincter dysfunction. Weaker legs were confirmed on neurological examination: hip flexion grade 2 bilaterally, knee flexion/extension grade 4 bilaterally, ankle dorsiflexion grade 2 on the right and 4 on the left, and hallux extension grade 2 on the right and 4 on the left; in addition, bilateral Babinski sign was confirmed. Deep tendon reflexes were now weak in lower limbs, and decreased pinprick and proprioceptive sensation (sensory level at L1) was revealed. Somatosensitive evoked potentials showed bilateral delayed cortical responses suggesting sensitive dorsal column lesion in the dorsal/lumbar

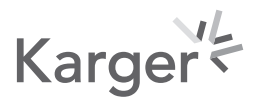



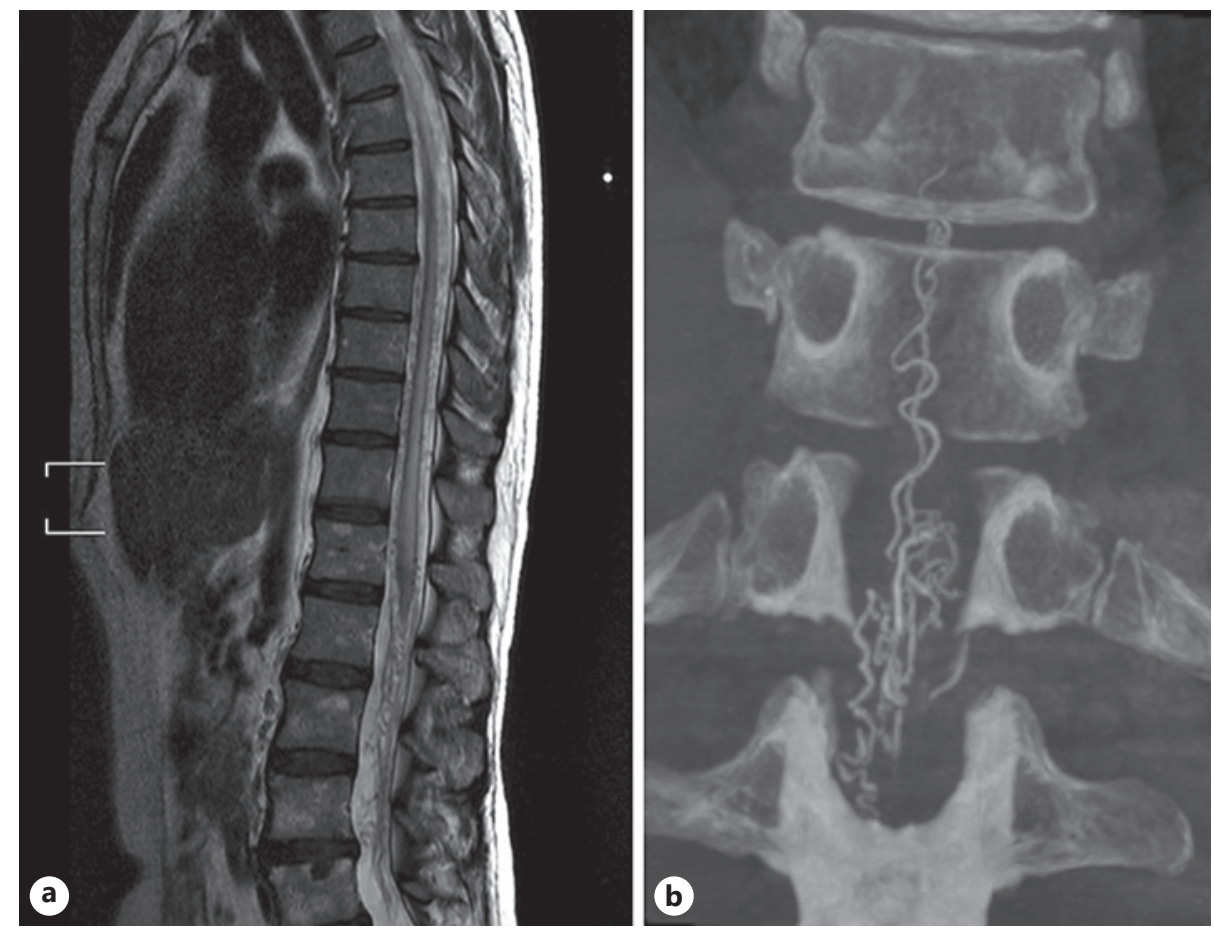

Fig. 1. a Sagittal view of T2 MRI showing spinal cord oedema from T7 to conus. b Conventional angiography and CT coronal reconstruction showing multiple perimedullary vessels suggestive of arteriovenous fistula.

region. Transcranial magnetic stimulation suggested mild pyramidal lesion to lower limbs (increased central conduction time). Changes on electromyography were similar to the ones previously reported.

Diagnosis of ALS became unlikely, and the patient accepted to repeat dorsal/lumbar MRI with sedation. This investigation showed extensive spinal cord oedema from T7 to the conus, with a long central hyperintensity on T2/STIR sequences and diffuse gadolinium enhancement (Fig. 1a). Additionally, it showed multiple perimedullary flow voids in the dorsal, lumbar, and conus region, suggestive of an arteriovenous fistula. Conventional angiography confirmed the diagnosis, revealing the fistula at L2-L3 level with the left L4 lumbar branch as the afferent artery (Fig. 1b). The patient is awaiting surgical treatment.

\section{Discussion/Conclusion}

The initial presentation of lower and upper motor neuron signs in lower limbs suggested ALS. However, the absence of signs indicating active denervation on electromyography [3] despite progressive lower limb weakness was not typical for patients with lumbo-sacral onset. In addition, later sensory deficits and sphincter dysfunction led to workup review.

Both diseases share demographic features, being more common in males around their 50s. Most SDAVF patients present both upper and lower motor neuron deficits [4], which makes this entity a mimic of ALS. SDAVF occurs in 5-10 persons per million [5], and most fistulas are located between T5 and L5 segments [1]. The arteriovenous shunt causes arterialization of the radicular vein, and perimedullary venous plexus suffers engorgement and hypertension, which leads to spinal cord oedema (congestive radiculomyelopathy) and ischaemia. The variable blood flow rate in the fistula and the number of occluded radicular 
veins cause fluctuation and heterogeneity of manifestations. The progression of the cord oedema is usually caudal to cranial because of lower drainage channel in the lower spine, explaining the stepwise progression. Initial symptoms are mild, nonspecific, and usually symmetric or asymmetric motor/sensory deficits ascending proximally. Sphincter dysfunction appears on late stages.

Over $80 \%$ of cases of SDAVF are clinically misdiagnosed in the beginning [1]. The gold standard for the diagnosis is spinal angiography, but MRI frequently shows cord oedema, enlarged pia flow voids around the cord, and gadolinium enhancement due to venous engorgement and disruption of the blood-brain barrier [5]. However, clinical signs of mild myelopathy may exist without imaging evidence in around 6\% of cases [1]. In the present case, as the disease continued to progress, appearance of new deficits was essential to convince the patient to repeat neuroimaging.

As arteriovenous fistulas are unlikely to spontaneously close, endovascular treatment or open surgery is needed. Bad prognostic signs for recovery are extensive cord oedema and enhancement, severe neurologic deficit, and sphincter dysfunction [5]. Delayed diagnosis may compromise the patient outcome.

This case shows how close follow-up and thorough investigation, especially in difficult cases, may be essential for the diagnosis of treatable conditions. Also, it brings to close attention a novel mimic of ALS, often misdiagnosed, which requires a high level of suspicion in the early course.

\section{Statement of Ethics}

The subject has given his written informed consent to publish his case including any accompanying images. The study is exempt from ethics committee approval because complete patient anonymity was guaranteed.

\section{Conflict of Interest Statement}

The authors have no conflicts of interest to declare.

\section{Funding Sources}

No funding was used.

\section{Author Contributions}

Mónica Santos contributed to resources and writing - original draft; Sofia Reimão contributed to investigation, resources, writing - review and editing, and supervision; Mamede de Carvalho contributed to investigation, writing - review and editing, and supervision.

\section{Data Availability Statement}

All data analysed during this study are included in this article. Further enquiries can be directed to the corresponding author. 


\section{References}

1 Donghai W, Ning Y, Peng Z, Shuo X, Xueen L, Peng Z, et al. The diagnosis of spinal dural arteriovenous fistulas. Spine. 2013;38(9):E546-53.

2 de Carvalho M, Dengler R, Eisen A, England JD, Kaji R, Kimura J, et al. Electrodiagnostic criteria for diagnosis of ALS. Clin Neurophysiol. 2008;119(3):497-503.

3 de Carvalho M, Bentes C, Evangelista T, Luis ML. Fibrillation and sharp-waves: do we need them to diagnose ALS? Amyotrophic lateral sclerosis and other motor neuron disorders. Amyotroph Lateral Scler Other Motor Neuron Disord. 1999;1(1):29-32.

4 Jellema K, Canta LR, Tijssen CC, van Rooij WJ, Koudstaal PJ, van Gijn J. Spinal dural arteriovenous fistulas: clinical features in 80 patients. J Neurol Neurosurg Psychiatry. 2003;74(10):1438-40.

5 Maimon S, Luckman Y, Strauss I. Spinal dural arteriovenous fistula: a review. Adv Tech Stand Neurosurg. 2016(43):111-37. 